\title{
TAILS IN GENERALIZED JACKSON NETWORKS WITH SUBEXPONENTIAL SERVICE-TIME DISTRIBUTIONS
}

\author{
FRANÇOIS BACCELLI, ${ }^{* *}$ INRIA-ENS \\ SERGUEI FOSS, ${ }^{* * *}$ Heriot-Watt University and Institute of Mathematics, Novosibirsk \\ MARC LELARGE, ${ }^{* * * * *}$ INRIA-ENS
}

\begin{abstract}
We give the exact asymptotics of the tail of the stationary maximal dater in generalized Jackson networks with subexponential service times. This maximal dater, which is an analogue of the workload in an isolated queue, gives the time taken to clear all customers present at some time $t$ when stopping all arrivals that take place later than $t$. We use the property that a large deviation of the maximal dater is caused by a single large service time at a single station at some time in the distant past of $t$, in conjunction with fluid limits of generalized Jackson networks, to derive the relevant asymptotics in closed form.
\end{abstract}

Keywords: Generalized Jackson network; subexponential random variable; heavy tail; integrated tail; Veraverbeke's theorem; fluid limit

2000 Mathematics Subject Classification: Primary 60K25

Secondary $62 \mathrm{G} 20$

\section{Introduction}

To the best of the authors' knowledge, the literature on generalized Jackson networks with heavy-tailed service times is limited to tandem queues. Exact asymptotics for these quantities were obtained in [4]. The present paper addresses the case of generalized Jackson networks of arbitrary topology. It focuses on a key state variable, namely the time to empty the network when stopping the arrival process, which has previously been used to determine the stability region of such networks [2], [3]. (This variable boils down to the virtual workload in an isolated queue or to the sojourn time for tandem queues.) The aim of the paper is to derive the exact asymptotics of the tail of this state variable in the stationary regime. The main ingredients for the derivation of this result are as follows.

- We require a generalization of the so-called 'single-big-event theorem', well known for isolated queues, to generalized Jackson networks of the sort we consider. This was established in [4]. In the GI/GI/1 queue, this theorem states that, in the case of subexponential service times, large workloads typically occur in the event that a single large service time has taken place in the distant past, while all the other service times were close to their means. Similarly, in generalized Jackson networks with subexponential

Received 5 January 2004; revision received 19 October 2004.

* Postal address: ENS-DI, 45 rue d'Ulm, 75005 Paris, France.

** Email address: francois.baccelli@ens.fr

*** Postal address: Department of Actuarial Mathematics and Statistics, Heriot-Watt University, Edinburgh EH14 4AS, UK. Email address: s.foss@ma.hw.ac.uk. Supported by INTAS grant 265 and by EPSRC grant R58765/01.

***** Email address: marc.lelarge@ens.fr 
service times, large maximal daters occur when a single large service time has taken place at one of the stations, while all the other service times are close to their means.

- We must identify the role played by fluid limits within the context of the single-big-event theorem for this class of networks.

- We must find the combination of these fluid limits and heavy-tailed calculus that allows us to derive the closed form formulae for the asymptotics.

Although this result sheds light on the way such a network experiences a deviation from its normal behaviour, it is in no way final, as the tail behaviour of other state variables, such as the stationary queue size, are still unknown. The derivation of the (more complex) asymptotic behaviour of these other state variables has already been obtained using a similar methodology in the particular case of tandem queues [4]. The extension of these queue-size asymptotics to generalized Jackson networks of arbitrary topology seems to require much more effort and will not be pursued in the present paper. The proposed method should, however, extend to other characteristics of stationary workload like, for instance, the sum of the residual service times of all customers present in the network at some given time.

The paper is structured as follows. In Section 2, we introduce generalized Jackson networks and show that the four assumptions required in order to use the single-big-event theorem (called (IA), (AA), (SE), and (H) in [4]) hold. The main result is then established in Section 3.

\subsection{Notation}

Here and later in the paper, for positive functions $f$ and $g$, the equivalence $f(x) \sim d g(x)$ with $d>0$ means that $f(x) / g(x) \rightarrow d$ as $x \rightarrow \infty$. By convention, the equivalence $f(x) \sim d g(x)$ with $d=0$ means that $f(x) / g(x) \rightarrow 0$ as $x \rightarrow \infty$; this will be written $f(x)=o(g(x))$. We will also use the notation $f(x)=\mathcal{O}(g(x))$ to mean that both $\limsup _{x} f(x) / g(x)<\infty$ and $\liminf _{x} f(x) / g(x)>0$.

In this paper, $\varepsilon(x)$ denotes a function such that $\varepsilon(x) \rightarrow 0$ as $x \rightarrow \infty$. The function $\varepsilon$ may vary from place to place; for example, we write

$$
\varepsilon(x)+\varepsilon(x)=\varepsilon(x), \quad \varepsilon(x)(1+\varepsilon(x))=\varepsilon(x),
$$

and so on. Similarly, we will write $\varepsilon(x, y)$ for $\varepsilon(x)+\varepsilon(y), \varepsilon(x) \varepsilon(y)$, etc.

\section{Generalized Jackson networks}

\subsection{General framework and sample-path construction of the maximal dater}

2.1.1. Service time and routeing sequences. Here, we recall the notation introduced in [2] to describe a generalized Jackson network with $K$ nodes. The networks we consider are characterized by the fact that service times and routeing decisions are associated with stations and not with customers. This means that the $j$ th service at station $k$ takes $\sigma_{j}^{(k)}$ units of time, where $\left\{\sigma_{j}^{(k)}\right\}_{j \geq 1}$ is a predefined sequence. In the same way, when this service is completed, the departing customer is sent to station $v_{j}^{(k)}$ (or leaves the network, if $v_{j}^{(k)}=K+1$ ) and is put at the end of the queue at this station, where $\left\{v_{j}^{(k)}\right\}_{j \geq 1}$ is also a predefined sequence, called the routeing sequence. The sequences $\left\{\sigma_{j}^{(k)}\right\}_{j \geq 1}$ and $\left\{v_{j}^{(k)}\right\}_{j \geq 1}$, where $k$ ranges over the set of stations, are called the driving sequences of the network. Node 0 models the external arrival of customers to the network and, therefore, the arrival time of the $j$ th customer in the network takes place at $\sigma_{1}^{(0)}+\cdots+\sigma_{j}^{(0)}$ and it joins the end of the queue at station $v_{j}^{(0)}$. Hence, $\sigma_{j}^{(0)}$ is the $j$ th interarrival time. 
The sample-path construction introduced here is that of [2]. The main interest of such a construction is that some monotonicity properties are preserved, as explained in [2]. As was shown in [4], these monotonicity properties are crucial for our asymptotics calculation.

A generalized Jackson network will be defined by

$$
\mathbf{J N}=\left\{\left\{\sigma_{j}^{(k)}\right\}_{j \geq 1},\left\{v_{j}^{(k)}\right\}_{j \geq 0}, n^{(k)}, 0 \leq k \leq K\right\},
$$

where $N=\left(n^{(0)}, n^{(1)}, \ldots, n^{(K)}\right)$ describes the initial condition. The interpretation is as follows: at time $t=0$, in node $i, i \neq 0$, there are $n^{(i)}$ customers, with service times $\sigma_{1}^{(i)}, \ldots, \sigma_{n^{(i)}}^{(i)}$ (if appropriate, $\sigma_{1}^{(i)}$ may be interpreted as a residual service time). On the other hand, the interpretation of $n^{(0)}$ (the $i=0$ case) is as follows.

- If $n^{(0)}=0$ there is no external arrival.

- If $\infty>n^{(0)} \geq 1$ then, for all $1 \leq j \leq n^{(0)}$, the arrival time of the $j$ th customer in the network takes place at $\sigma_{1}^{(0)}+\cdots+\bar{\sigma}_{j}^{(0)}$. Note that, in this case, there may be a finite number of customers passing through a given station, so that the network is actually well defined once a finite sequence of routeing decisions and service times is given for this station.

- If $n^{(0)}=\infty$ then when taking, for instance, the sequence $\left\{\sigma_{j}^{(0)}\right\}_{j \geq 1}$ to be independent and identically distributed (i.i.d.), the arrival process is a renewal process.

2.1.2. Euler route, Euler network. Consider a route $\boldsymbol{r}=\left(r_{1}, \ldots, r_{\phi}\right)$ with $1 \leq r_{i} \leq K$ for $i=2, \ldots, \phi-1$. Such a route is successful if $r_{1}=0$ and $r_{\phi}=K+1$. To such a route, we associate a routeing sequence $v=\left(v^{(0)}, \ldots, v^{(K)}\right)$ as follows (here, ' $\oplus$ ' means concatenation and $\varnothing$ denotes the empty sequence).

\section{Procedure $(\boldsymbol{r})$ :}

$-1-\quad$ for $k=0, \ldots, K$ do

$$
\begin{aligned}
& v^{(k)}:=\varnothing ; \\
& \phi^{(k)}:=0 ;
\end{aligned}
$$

od

$-2-\quad$ for $i=1, \ldots, \phi-1$ do

$$
\begin{aligned}
& v^{\left(r_{i}\right)}:=v^{\left(r_{i}\right)} \oplus r_{i+1} ; \\
& \phi^{\left(r_{i}\right)}:=\phi^{\left(r_{i}\right)}+1 ;
\end{aligned}
$$

od

Note that $\phi^{(j)}$ is the number of visits to node $j$ in such a route.

A simple Euler network is a generalized Jackson network $E=\{\sigma, v, N\}$, with $N=$ $(1,0, \ldots, 0)=1$, such that the routeing sequence $v=\left\{v_{i}^{(k)}\right\}_{i=1}^{\phi^{(k)}}$ is generated by a successful route and such that $\sigma=\left\{\sigma_{i}^{(k)}\right\}_{i=1}^{\phi^{(k)}}$ is a sequence of real-valued nonnegative numbers, representing service times.

Consider a sequence of simple Euler networks, say $\{E(n)\}_{n=-\infty}^{0}$, where $E(n)=\{\sigma(n)$, $v(n), 1\}$. For $m \leq n \leq 0$, we define $\sigma_{[m, n]}$ and $\nu_{[m, n]}$ to be the concatenation of $\{\sigma(k)\}_{m \leq k \leq n}$ 
and $\{v(k)\}_{m \leq k \leq n}$, and then define the following composed generalized Jackson network, with $N_{[m, n]}=(n-m+1,0, \ldots, 0)$ :

$$
\mathbf{J N}_{[m, n]}=\left\{\sigma_{[m, n]}, v_{[m, n]}, N_{[m, n]}\right\}
$$

2.1.3. Maximal dater. As proved in [2], for all possible values of $v(p)$ and $\sigma(p)$ in the simple Euler networks, and for all integers $m \leq n$, the composed network $\mathbf{J} \mathbf{N}_{[m, n]}$ stays empty forever after some finite time. We denote by $X_{[m, n]}$ the time taken to empty $\mathbf{J N}_{[m, n]}$ forever and, by

$$
Z_{[m, n]}=X_{[m, n]}-\sum_{i=1}^{n-m+1} \sigma_{[m, n], i}^{(0)},
$$

the associated maximal dater. The sequence $Z_{[-n, 0]}$ is an increasing sequence. We define the maximal dater of the generalized Jackson network $\mathbf{J N}=\{\sigma, v, N\}$, where $\sigma$ and $v$ are the infinite concatenations of the $\{\sigma(n)\}_{n}$ and $\{v(n)\}_{n}$, and $N=(\infty, 0, \ldots, 0)$, by

$$
Z=\lim _{n \rightarrow \infty} Z_{[-n, 0]}
$$

To all generalized Jackson networks $\mathbf{J N}_{[m, n]}$, we also associate the generalized Jackson network $\mathbf{J N}_{[m, n]}(0)$, in which the driving sequences are the same as in the original network except for the sequence $\left\{\sigma_{j}^{(0)}\right\}$, which is now $\sigma_{j}^{(0)}=0$ for all $j$. Similarly, we define $Z_{[m, n]}(0)$ to be the time taken to empty the generalized Jackson network $\mathbf{J} \mathbf{N}_{[m, n]}(0)$.

Let

$$
Y_{i}^{(k)}=\sum_{j=1}^{\phi^{(k)}(i)} \sigma_{j}^{(k)}(i)
$$

be the total load brought by (external) customer $i$ to station $k$. In this expression, $\phi^{(k)}(i)$ is the number of visits of customer $i$ to station $k$ if he is alone in the network, and $\sigma_{j}^{(k)}(i)$ denotes his service time at this station. Note that

$$
\begin{aligned}
& Z_{i}:=Z_{[i, i]}=Y_{i}^{(1)}+\cdots+Y_{i}^{(K)} \quad \text { for all } i, \\
& Z_{[n, 0]}(0) \geq \max _{j=1, \ldots, K} \sum_{i=n}^{0} Y_{i}^{(j)} \text { for all } n \leq 0 .
\end{aligned}
$$

In addition, Lemma 4 of [3] implies that

$$
\lim _{n \rightarrow \infty} \frac{Z_{[-n, 0]}(0)}{n}=: b=\max _{1 \leq k \leq K} \mathrm{E}\left[Y_{1}^{(k)}\right] \quad \text { almost surely. }
$$

\subsection{Stochastic assumptions}

Assumption 2.1. (Independence of routeing and service times.) The sequences $\left\{v^{(k)}\right\}$ and $\left\{\sigma^{\left(k^{\prime}\right)}\right\}$ are mutually independent, for $k$ and $k^{\prime}$ ranging over the set of stations.

Assumption 2.2. (Independence of service times.) We assume that the service times are independent for different stations and, in each station, i.i.d. with finite mean: $\mathrm{E}\left[\sigma^{(j)}\right]=1 / \mu^{(j)}>0$ for all $1 \leq j \leq K$. 
Assumption 2.3. (Routeing.) We assume that each of the successful routes used to build $v$ is obtained by a Markov chain on the state space $\{0,1, \ldots, K, K+1\}$, with transition matrix

$$
\boldsymbol{R}=\left(\begin{array}{cccccc}
0 & r_{0,1} & r_{0,2} & \cdots & r_{0, K} & 0 \\
0 & r_{1,1} & r_{1,2} & \cdots & r_{1, K} & r_{1, K+1} \\
0 & r_{2,1} & r_{2,2} & \cdots & r_{2, K} & r_{2, K+1} \\
\vdots & \vdots & \vdots & & \vdots & \vdots \\
0 & r_{K, 1} & r_{K, 2} & \cdots & r_{K, K} & r_{K, K+1} \\
0 & 0 & 0 & \cdots & 0 & 1
\end{array}\right)
$$

This is equivalent to assuming that the routeing decisions $\left\{v_{j}^{(k)}\right\}$ at station $k$ are i.i.d. in $j$, independent of everything else, and such that the routeing decision selects station $i$ with probability $\mathrm{P}\left[v^{(k)}=i\right]=r_{k, i}$. The fact that the routes built with this Markovian procedure are successful implies that state $K+1$ is the only absorbing state of this chain and that all other states are transient; we then have the very same Markovian routeing assumptions as in (exponential) Jackson networks. More generally, when denoting by $\mathrm{E}_{k}$ the law of the chain with initial condition $k$ and, by $V_{j}$, the number of visits of this absorbing chain to state $j$, we define

$$
\mathrm{E}_{0}\left[V_{k}\right]=\pi_{k}, \quad \mathrm{P}_{0}\left[V_{k} \geq 1\right]=p_{k}, \quad \mathrm{E}_{k}\left[V_{j}\right]=\pi_{k, j} .
$$

We will use the following notation:

$$
b_{j}=\frac{\pi_{j}}{\mu^{(j)}}, \quad b_{j, i}=\frac{\pi_{j, i}}{\mu^{(i)}}, \quad B_{j}=\max _{i} b_{j, i} .
$$

With this notation, we have $b=\max _{i} \pi_{i} / \mu^{(i)}=\max _{i} b_{i}$. Let $\lambda^{-1}=\mathrm{E}\left[\sigma^{0}\right]=a$. Throughout this paper we will assume that the following stability condition holds:

$$
\lambda b<1 .
$$

Theorem 13 of [2] applies, meaning that if $\lambda b<1$, then $Z<\infty$ almost surely; conversely, if $\lambda b>1$ then $Z=\infty$ almost surely.

Example 2.1. As an example, we will consider a network with the following routeing matrix:

$$
\boldsymbol{R}=\left\{\begin{array}{cccc}
0 & 1 & 0 & 0 \\
0 & 0 & p & 1-p \\
0 & q & 0 & 1-q \\
0 & 0 & 0 & 1
\end{array}\right\}
$$

In this case, we have

$$
\pi_{1}=1+q \pi_{2}, \quad \pi_{2}=p \pi_{1},
$$

which imply that

$$
\pi_{1}=\frac{1}{1-p q}, \quad \pi_{2}=\frac{p}{1-p q} .
$$

Similarly, we have

$$
\begin{array}{ll}
\pi_{1,1}=\pi_{1}, & \pi_{1,2}=\pi_{2}, \\
\pi_{2,1}=\frac{q}{1-p q}, & \pi_{2,2}=\frac{1}{1-p q},
\end{array}
$$


and

$$
p_{1}=1, \quad p_{2}=p
$$

Hence, we have

$$
b=B_{1}=\frac{1}{1-p q} \max \left\{\frac{1}{\mu^{(1)}}, \frac{p}{\mu^{(2)}}\right\} \quad \text { and } \quad B_{2}=\frac{1}{1-p q} \max \left\{\frac{q}{\mu^{(1)}}, \frac{1}{\mu^{(2)}}\right\} .
$$

In particular, for $p=1$ and $q=0$, we are dealing with the case of queues in tandem and we have $b=B_{1}=\max \left(1 / \mu^{(1)}, 1 / \mu^{(2)}\right)$ and $B_{2}=1 / \mu^{(2)}$.

We now recall some definitions.

Definition 2.1. A distribution function $F$ on $\mathbb{R}_{+}$(with $\bar{F}=1-F$ ) is long tailed if, for any $y>0$,

$$
\bar{F}(x+y) \sim \bar{F}(x) \text { as } x \rightarrow \infty .
$$

We introduce a proper subset of the class of long-tailed distributions, namely the class of subexponential distributions, denoted by $\&$.

Definition 2.2. A distribution function $F$ on $\mathbb{R}_{+}$is called subexponential if $\overline{F^{* 2}}(x) \sim 2 \bar{F}(x)$, where $\overline{F^{* 2}}(x)$ is the tail of the two-fold convolution of $F$.

Definition 2.3. A positive measurable function $f$ on $[0, \infty)$ is called regularly varying with index $\alpha \in \mathbb{R}$ (denoted $f \in \mathcal{R}(\alpha)$ ) if

$$
\lim _{x \rightarrow \infty} \frac{f(t x)}{f(x)}=t^{\alpha} \quad \text { for all } t>0 .
$$

Definition 2.4. A positive measurable function $h$ on $[0, \infty)$ is called rapidly varying (denoted $h \in \mathcal{R}(-\infty))$ if

$$
\lim _{x \rightarrow \infty} \frac{h(t x)}{h(x)}=0 \quad \text { for all } t>1 .
$$

For example, Weibull or lognormal random variables have tail distributions that are rapidly varying. For more on the matter, see [5] and [6].

Assumption 2.4. (Subexponentiality of service times.) For a distribution function $F$ on the positive real line, with finite first moment $M=\int_{0}^{\infty} \bar{F}(u) \mathrm{d} u, \bar{F}(u)=1-F(u)$ denotes the tail of $F$ and $F^{s}$ the integrated tail distribution, given by

$$
F^{s}(x)=1-\min \left\{1, \int_{x}^{\infty} \bar{F}(u) \mathrm{d} u\right\}=: 1-\bar{F}^{s}(x) .
$$

The assumptions concerning service times are the following. There exists a distribution function F on $\mathbb{R}_{+}$such that

1. F is subexponential, with finite first moment $M$;

2. the integrated distribution $F^{s}$ is subexponential; and

3. the following equivalence holds when $x$ tends to $\infty$ :

$$
\mathrm{P}\left[\sigma_{1}^{(k)}>x\right] \sim c^{(k)} \bar{F}(x)
$$

for all $k=1, \ldots, K$, with $\sum_{k=1}^{K} c^{(k)}=: c>0$. 


\subsection{Main result}

We first introduce some notation; the intuitive meanings of these quantities will be given later on. Let $f^{j}(\sigma, n)$ be the following piecewise-linear function of the nonnegative real numbers $\sigma$ and $n$ :

$$
f^{j}(\sigma, n)=\mathbf{1}_{\{\sigma>n a\}}\left(\sigma-n a+n p_{j} B_{j}\right)+\mathbf{1}_{\{\sigma \leq n a\}} \max _{k}\left\{p_{j} b_{j, k} \frac{\sigma}{a}+\left(\frac{b_{k}}{a}-1\right)(n a-\sigma)\right\}^{+} .
$$

In addition, for all positive real numbers $x$ and all $j=1, \ldots, K$, let $\Delta^{j}(x)$ be the following domain:

$$
\Delta^{j}(x)=\left\{(\sigma, t) \in \mathbb{R}_{+}^{2}: f^{j}(\sigma, t)>x\right\} .
$$

Remark 2.1. We may rewrite the function $f^{j}$ as

$$
f^{j}(\sigma, n)=\left\{\sigma-n a+\max _{k}\left\{n b_{k}-\frac{\sigma}{a}\left(b_{k}-p_{j} b_{j, k}\right), n p_{j} b_{j, k}\right\}\right\}^{+} .
$$

This is due to the fact that $p_{j} b_{j, k} \leq b_{k}$. In particular, we see that

$$
f^{j}(\sigma, n) \geq \sigma-n a-n p_{j} B_{j} .
$$

Example 2.2. Continuing Example 2.1, we have

$$
\begin{aligned}
& f^{1}(\sigma, n)=\left\{\sigma-n a+\frac{n}{1-p q} \max \left\{\frac{1}{\mu^{(1)}}, \frac{p}{\mu^{(2)}}\right\}\right\}^{+}, \\
& f^{2}(\sigma, n)=\left\{\sigma-n a+\max \left\{\frac{n p q}{(1-p q) \mu^{(1)}}, \frac{n p}{(1-p q) \mu^{(2)}}, \frac{n}{(1-p q) \mu^{(1)}}-\frac{\sigma}{a \mu^{(1)}}\right\}\right\}^{+} .
\end{aligned}
$$

In the specific case of queues in tandem ( $p=1$ and $q=0)$, these equations reduce to

$$
\begin{aligned}
& f^{1}(\sigma, n)=\left\{\sigma-n a+n \max \left\{\frac{1}{\mu^{(1)}}, \frac{1}{\mu^{(2)}}\right\}\right\}^{+}, \\
& f^{2}(\sigma, n)=\left\{\sigma-n a+\frac{n}{\mu^{(2)}}\right\}^{+},
\end{aligned}
$$

and the corresponding domains are

$$
\begin{aligned}
& \Delta^{1}(x)=\left\{\sigma>x+t\left(a-\max \left\{\frac{1}{\mu^{(1)}}, \frac{1}{\mu^{(2)}}\right\}\right)\right\}, \\
& \Delta^{2}(x)=\left\{\sigma>x+t\left(a-\frac{1}{\mu^{(2)}}\right)\right\} .
\end{aligned}
$$

Theorem 2.1. Consider a stable generalized Jackson network with subexponential servicetime distributions satisfying Assumptions 2.1-2.4. Let $Z$ denote its stationary maximal dater at customer arrivals (see Section 2.1 for a formal definition of $Z$ ). When $x \rightarrow \infty$,

$$
\mathrm{P}[Z>x] \sim \sum_{j=1}^{K} \pi_{j} \iint_{(\sigma, t) \in \Delta^{j}(x)} \mathrm{P}\left[\sigma^{(j)} \in \mathrm{d} \sigma\right] \mathrm{d} t .
$$


This equation may be rewritten with the constants $\left\{\alpha_{i}^{j}, \beta_{i}^{j}, \gamma_{i}^{j}\right\}_{0 \leq i \leq l}$, which will be calculated in Lemma 3.3 below, as

$$
\mathrm{P}[Z>x] \sim \sum_{j=1}^{K} \pi_{j}\left\{\sum_{i=0}^{l} \sum_{\alpha_{i}^{j} x \leq n<\alpha_{i+1}^{j}} \mathrm{P}\left[\sigma^{(j)}>\frac{x}{\beta_{i}^{j}}+n \gamma_{i}^{j}\right]\right\}
$$

or, using $\delta_{i}^{j}=1 / \beta_{i}^{j}+\alpha_{i}^{j} \gamma_{i}^{j}$ and $d^{(j)}=\pi_{j} c^{(j)}$, as

$$
\mathrm{P}[Z>x] \sim \sum_{j=1}^{K} d^{(j)}\left\{\sum_{i=0}^{l} \frac{1}{\gamma_{i}^{j}}\left[\bar{F}^{s}\left(\delta_{i}^{j} x\right)-\bar{F}^{s}\left(\delta_{i+1}^{j} x\right)\right]\right\} .
$$

The following statements then hold, as $x$ tends to $\infty$.

1. If $\bar{F}^{s} \in \mathcal{R}(-\alpha)$, with $\alpha>0$, we can rewrite (2.10) as

$$
\frac{\mathrm{P}[Z>x]}{\bar{F}^{s}(x)} \rightarrow \sum_{j=1}^{K} d^{(j)}\left\{\sum_{i=0}^{l} \frac{1}{\gamma_{i}^{j}}\left[\left(\delta_{i}^{j}\right)^{-\alpha}-\left(\delta_{i+1}^{j}\right)^{-\alpha}\right]\right\} .
$$

2. If $\bar{F}^{s} \in \mathcal{R}(-\infty)$ then

$$
\frac{\mathrm{P}[Z>x]}{\bar{F}^{s}(x)} \rightarrow \sum_{j=1}^{K} \frac{d^{(j)}}{a-p_{j} B_{j}} .
$$

Remark 2.2. In view of (2.6), we see that $\left\{\sigma>x+t\left(a-p_{j} B_{j}\right)\right\} \subset \Delta^{(j)}(x)$ and, hence,

$$
\mathrm{P}[Z>x] \geq \sum_{j=1}^{K} \pi_{j} \sum_{n \geq 0} \mathrm{P}\left[\sigma^{(j)}>x+n\left(a-p_{j} B_{j}\right)\right] \sim \sum_{j=1}^{K} \frac{d^{(j)}}{a-p_{j} B_{j}} \bar{F}^{s}(x) .
$$

Hence, in general, the asymptotics described in (2.11) are a lower bound. In the rapidly varying case, this lower bound is tight. In the regularly varying case, the complete shape of the domain must be taken into consideration and, indeed, the part $\Delta^{j}(x) \backslash\left\{\sigma>x+t\left(a-p_{j} B_{j}\right)\right\}$ is no longer negligible, due to the scaling property of regularly varying functions. More insights into the shape of the domain will be given in Section 3.3.

Example 2.3. In the specific case of queues in tandem, from (2.7) and (2.8) we see that (2.9) reduces to

$\mathrm{P}[Z>x] \sim \sum_{n \geq 0} \mathrm{P}\left[\sigma^{(1)}>x+n\left(a-\max \left\{\frac{1}{\mu^{(1)}}, \frac{1}{\mu^{(2)}}\right\}\right)\right]+\sum_{n \geq 0} \mathrm{P}\left[\sigma^{(2)}>x+n\left(a-\frac{1}{\mu^{(2)}}\right)\right]$,

which corresponds to the exact asymptotics of Theorem 9 of [4].

\subsection{Technical conditions}

The properties (IA) and (AA) of [4] are as follows.

(IA) The sequence of simple Euler networks $\{E(n)\}_{n=0}^{-\infty}$ consists of i.i.d. random variables. 
(AA) The random variables $\left\{Y_{i}^{(k)}\right\}$ are independent of the interarrival times and such that the sequence of random vectors $\left\{\left(Y_{i}^{(1)}, \ldots, Y_{i}^{(K)}\right)\right\}_{i \in \mathbb{N}}$ is i.i.d. (general dependences between the components of the vector $\left(Y_{i}^{(1)}, \ldots, Y_{i}^{(K)}\right)$ are allowed).

Under our Assumptions 2.1-2.3, both of these properties hold.

Under Assumption 2.1, the variable $Z$ associated to $\mathbf{J N}=\{\sigma, v, N\}$ represents the stationary maximal dater of the generalized Jackson network, namely the time that it would take, in steady state, to clear the workload of all customers present in the system, when stopping future arrivals.

The assumptions (SE) and (H) of [4] are as follows.

(SE) For all $k=1, \ldots, K$,

$$
\mathrm{P}\left[Y_{1}^{(k)}>x\right] \sim \pi^{k} \mathrm{P}\left[\sigma^{(k)}>x\right] \sim d^{(k)} \bar{F}(x),
$$

with $d^{(k)}=c^{(k)} \pi_{k}$. Thus, $d:=\sum_{k} d^{(k)}>0$.

(H) We have

$$
\mathrm{P}\left[\sum_{k=1}^{K} Y_{1}^{(k)}>x\right] \sim \mathrm{P}\left[\max _{1 \leq k \leq K} Y_{1}^{(k)}>x\right] \sim \sum_{k=1}^{K} \mathrm{P}\left[Y_{1}^{(k)}>x\right] \sim d \bar{F}(x) .
$$

(See Sections 4.4.2 and 7.2 of [4].) Under our Assumption 2.4, both of these assumptions are satisfied. Also under Assumption 2.4, there exists a nondecreasing, integer-valued function $N_{x}$ that tends to infinity as $x$ tends to infinity and is such that, for all finite real numbers $b$,

$$
\sum_{n=0}^{N_{x}} \bar{F}(x+n b)=o\left(\bar{F}^{s}(x)\right), \quad x \rightarrow \infty
$$

(see Section 4.1.2 of [4]).

\section{Exact tail asymptotics}

\subsection{Single-big-event theorem}

As already mentioned, one of the tools we will use within this setting is the 'single-big-event theorem' for generalized Jackson networks. More precisely, Theorems 7 and 8 of [4] give the following result.

Proposition 3.1. Let $Z$ be the stationary maximal dater of the generalized Jackson network defined in (2.1). For any $x$ and for $j=1, \ldots, r$, let $\left\{K_{n, x}^{j}\right\}$ be a sequence of events such that,

1. for any $n$ and $j$, the event $K_{n, x}^{j}$ and the random variable $Y_{-n}^{(j)}=\sum_{k=1}^{\phi^{(j)}(-n)} \sigma_{k}^{(j)}(-n)$ are independent; and

2. for any $j, \mathrm{P}\left[K_{n, x}^{j}\right] \rightarrow 1$, uniformly in $n \geq N_{x}$, as $x \rightarrow \infty$.

For all sequences $\varepsilon_{n} \rightarrow 0$, we write $x_{n}=x+n\left(a-b+\varepsilon_{n}\right)$. Then, as $x \rightarrow \infty$,

$$
\mathrm{P}[Z>x] \sim \sum_{j=1}^{K} \sum_{n \geq N_{x}} \mathrm{P}\left[Z>x, Y_{-n}^{(j)}>x_{n}, K_{n, x}^{j}\right]
$$

and $\mathrm{P}[Z>x]=\mathcal{O}\left(\bar{F}^{s}(x)\right)$. 
Proposition 3.1 leads to the following, more convenient, result.

Corollary 3.1. Take any sequence of events $\left\{K_{n}^{j}\right\}$ such that, for any $j, K_{n}^{j}$ and the random variable $Y_{-n}^{(j)}$ are independent, and $\mathrm{P}\left(K_{n}^{j}\right) \rightarrow 1$ as $n \rightarrow \infty$. Take $z_{x} \rightarrow \infty$, with $z_{x}=o(x)$, such that $\bar{F}^{s}\left(x \pm z_{x}\right) \sim \bar{F}^{s}(x)$, and let

$$
G(x)=\sum_{j=1}^{K} \sum_{n \geq N_{x}} \mathrm{P}\left[Z_{[-n, 0]}>x, K_{n}^{j}, Y_{-n}^{(j)}>x_{n}, \phi^{(j)}(-n) \leq L\right] .
$$

Then we have

$$
(1+\varepsilon(x)) G(x) \leq \mathrm{P}[Z>x] \leq(1+\varepsilon(x)) G\left(x-z_{x}\right)+\varepsilon(L, x) \bar{F}^{s}(x) .
$$

Furthermore, if $G$ is long tailed we have

$$
\mathrm{P}[Z>x] \sim G(x) \text { as } x \rightarrow \infty .
$$

The proof can be found in Appendix A.

\subsection{Fluid limit}

We have to find sequences of events $\left\{K_{n}^{j}\right\}$ allowing us to calculate the sum

$$
\sum_{n \geq N_{x}} \mathrm{P}\left[Z_{[-n, 0]}>x, K_{n}^{j}, Y_{-n}^{(j)}>x_{n}, \phi^{(j)}(-n) \leq L\right],
$$

where, as above, $x_{n}=x+n\left(a-b+\varepsilon_{n}\right)$. The events in question will be based on the piecewiselinear functions $f^{j}(\sigma, n)$ defined in (2.4). Let us describe the intuitive reason for introducing this function. Assume that the 'big' service time is equal to $\sigma$ and takes place at station $j$ within the set of service times of the simple Euler network $E(-n)$. Let us look at the maximal dater $Z_{[-n, 0]}$ in the fluid scale suggested by the almost-sure limit of (2.2).

- If $\sigma>n a$ then the number of customers queued at station $j$ at time $\sigma$ is of order $n p_{j}$, whereas the number of customers at the other stations is small. So, according to (2.2), the time taken to empty the network, from time $\sigma$ on, should be of order $n p_{j} B_{j}$. Hence, in this case, the maximal dater in question should indeed be of order $f^{j}(\sigma, n)$.

- If $\sigma<n a$ then, at time $\sigma$, the number of customers blocked at station $j$ is of order $p_{j} \sigma / a$, and the other stations have few customers; from time $\sigma$ to the time of the last arrival (which is of order $n a$ ), station $k$ has to serve the approximate load $p_{j}(\sigma / a) b_{j, k}$ generated by these blocked customers plus the load $(n a-\sigma) b_{k} / a$ generated by the external arrivals in the time interval from $\sigma$ to the last arrival. In this time interval, the service capacity is of order $(n a-\sigma)$. Hence, the maximal dater should again be of order $f^{j}(\sigma, n)$.

Particularly for the last case, a more thorough understanding of the fluid limit is clearly necessary. This is provided by the results of [7]. We now return to a more rigorous treatment.

Consider a generalized Jackson network built from the i.i.d. sequence of simple Euler networks $\{E(k)\}$. To all simple Euler networks $E$ and all positive integers $n$, we associate the network $\mathbf{J N}^{n}(E)$ with input $\{\tilde{E}(k)\}_{k=-n}^{\infty}$, where $\tilde{E}(k)=E(k)$ for all $k>-n$ and $\tilde{E}(-n)=E$. 
We allow for a specific initial condition for $k=-n$ and then take the original sequence. That is, if we denote by $\sigma^{(k), n}$ and $v^{(k), n}$ the concatenations

$$
\left(\left\{\sigma^{(k)}(E)\right\},\left\{\sigma^{(k)}(-n+1)\right\}, \ldots,\left\{\sigma^{(k)}(0)\right\}, \ldots\right)
$$

and

$$
\left(\left\{v^{(k)}(E)\right\},\left\{v^{(k)}(-n+1)\right\}, \ldots,\left\{v^{(k)}(0)\right\}, \ldots\right)
$$

respectively, then

$$
\mathbf{J N}^{n}(E)=\left\{\sigma^{n}(E), v^{n}(E), N^{n}\right\}, \quad \text { with } N^{n}=(n, 0, \ldots, 0) .
$$

The maximal dater of order $[-n, 0]$ in this network will be denoted by $\tilde{Z}^{n}(E)$; of course $\tilde{Z}^{n}(E(n))=Z_{[-n, 0]}$. More generally, we will add the superscript ' $n$ ' to any other function associated to the network $\mathbf{J N}^{n}(E)$.

For all simple Euler networks $E=(\sigma, v, 1)$, let $Y^{(j)}(E)=\sum_{u=1}^{\phi^{(j)}} \sigma_{u}^{(j)}$. We are now in a position to state the main result pertaining to the fluid limit. Let $\varepsilon_{n}$ and $z_{n}$ be some sequences of positive real numbers. We define

$$
\begin{aligned}
\boldsymbol{U}^{j}(n) & =\left\{E \text { is a simple Euler network such that } Y^{(k)}(E) \leq z_{n} \text { for all } k \neq j\right\}, \\
\boldsymbol{V}^{j}(n) & =\left\{E \in \boldsymbol{U}^{j}(n), Y^{(j)}(E) \geq n(a-b), \phi^{(j)} \leq L\right\}, \\
K_{n}^{j} & =\left\{\sup _{E \in \boldsymbol{V}^{j}(n)}\left|\frac{\tilde{Z}^{n}(E)-f^{j}\left(Y^{(j)}(E), n\right)}{n}\right| \leq \varepsilon_{n}\right\}, \\
\bar{K}_{n}^{j} & =K_{n}^{j} \cap\left\{E(-n) \in \boldsymbol{U}^{j}(n)\right\} .
\end{aligned}
$$

We first recall a result that derives directly from Proposition 5.1 and Remark 5.1 of [7].

Proposition 3.2. Under the previous assumptions, there exists a sequence $z_{n} \rightarrow \infty$, with $z_{n} / n \rightarrow 0$, such that

$$
\sup _{E \in V^{j}(n)}\left|\frac{\tilde{Z}^{n}(E)-f^{j}\left(Y^{(j)}(E), n\right)}{n}\right| \stackrel{n \rightarrow \infty}{\longrightarrow} 0 \text { almost surely. }
$$

Lemma 3.1. We have, as $x \rightarrow \infty$,

$$
G(x)=\sum_{j=1}^{K} \sum_{n \geq N_{x}} \mathrm{P}\left[Z_{[-n, 0]}>x, \bar{K}_{n}^{j}, Y_{-n}^{(j)}>x_{n}, \phi^{(j)}(-n) \leq L\right]+o\left(\bar{F}^{s}(x)\right) .
$$

Proof. Note that, owing to Proposition 3.2, the sequence of events $\left\{K_{n}^{j}\right\}$ satisfies the assumptions of Corollary 3.1. Since $\bar{K}_{n}^{j} \subset K_{n}^{j}$, we have

$$
G(x) \geq \sum_{j=1}^{K} \sum_{n \geq N_{x}} \mathrm{P}\left[Z_{[-n, 0]}>x, \bar{K}_{n}^{j}, Y_{-n}^{(j)}>x_{n}, \phi^{(j)}(-n) \leq L\right] .
$$

To find an upper bound, note that

$$
\begin{aligned}
& \mathrm{P}\left[Z_{[-n, 0]}>x, K_{n}^{j}, Y_{-n}^{(j)}>x_{n}, \phi^{(j)}(-n) \leq L\right] \\
& \leq \mathrm{P}\left[Z_{[-n, 0]}>x, \bar{K}_{n}^{j}, Y_{-n}^{(j)}>x_{n}, \phi^{(j)}(-n) \leq L\right] \\
& \quad+\mathrm{P}\left[Y_{-n}^{(j)}>x_{n}, \sum_{i \neq j} Y_{-n}^{(i)}>z_{n}\right] .
\end{aligned}
$$


Therefore, Lemma 3.1 will follow from our next result.

\section{Lemma 3.2.}

$$
\sum_{n \geq N_{x}} \mathrm{P}\left[Y_{-n}^{(j)}>x_{n}, \sum_{i \neq j} Y_{-n}^{(i)}>z_{n}\right]=o\left(\bar{F}^{s}(x)\right) .
$$

Proof. We use the same notation as in Appendix 7.2 of [2]. We have (with $z_{x}=z_{N_{x}}$ )

$$
\begin{aligned}
\frac{\mathrm{P}\left[Y_{-n}^{(j)}>x_{n}, \sum_{i \neq j} Y_{-n}^{(i)}>z_{n} \mid v\right]}{\bar{F}\left(x_{n}\right) \bar{F}\left(z_{x}\right)} & =\frac{\mathrm{P}\left[Y_{-n}^{(j)}>x_{n} \mid v\right]}{\bar{F}\left(x_{n}\right)} \frac{\mathrm{P}\left[\sum_{i \neq j} Y_{-n}^{(i)}>z_{n} \mid v\right]}{\bar{F}\left(z_{x}\right)} \\
& \leq \tilde{d}_{\nu}^{(j)} \sum_{i \neq j} \tilde{d}_{\nu}^{(i)} .
\end{aligned}
$$

Taking the sum over $n$ and then the expectation, we have

$$
\sum_{n \geq N_{x}} \mathrm{P}\left[Y_{-n}^{(j)}>x_{n}, \sum_{i \neq j} Y_{-n}^{(i)}>z_{n}\right] \leq \mathrm{E}\left[\tilde{d}_{\nu}^{(j)} \sum_{i \neq j} \tilde{d}_{\nu}^{(i)}\right] \bar{F}\left(z_{x}\right) \sum_{n \geq N_{x}} \bar{F}\left(x_{n}\right),
$$

which gives the result, completing the proofs of Lemma 3.2 and, hence, Lemma 3.1.

\subsection{Computation of the exact asymptotics}

In this section, we show that we can calculate the sum in (3.2). Its use in Corollary 3.1 and Lemma 3.1 will give the exact asymptotics of $\mathrm{P}[Z>x]$ in Theorem 2.1. Before stating this result, we must introduce some additional notation.

In the event $\bar{K}_{n}^{j} \cap\left\{Y_{-n}^{(j)}>x_{n}, \phi^{(j)}(-n) \leq L\right\}$, we have

$$
Z_{[-n, 0]}=f^{j}\left(Y_{-n}^{(j)}, n\right)+n \eta_{n}, \quad \text { with } \eta_{n} \text { a random variable such that }\left|\eta_{n}\right| \leq \varepsilon_{n} .
$$

Then, $\left\{Z_{[-n, 0]}>x\right\}=\left\{f^{j}\left(Y_{-n}^{(j)}, n\right)>x-n \eta_{n}\right\}$. In order to prove the equivalence (2.9), we will first give an explicit form for the domains $\Delta^{j}$ defined in (2.5).

Lemma 3.3. There exist constants $\left\{\alpha_{i}^{j}, \beta_{i}^{j}, \gamma_{i}^{j}\right\}_{0 \leq i \leq l}$ (given in closed form, in the proof of the present lemma, as functions of the quantities $p_{j}$ and $b_{j, k}$ defined in Section 2.2), with $0=\alpha_{0}^{j} \leq \alpha_{1}^{j} \leq \cdots \leq \alpha_{l}^{j}$, such that

$$
\Delta^{j}(z)=\bigcup_{i=0}^{l}\left\{\alpha_{i}^{j} z \leq t<\alpha_{i+1}^{j} z, \sigma>\frac{z}{\beta_{i}^{j}}+t \gamma_{i}^{j}\right\}
$$

with the convention that $\alpha_{l+1}^{j}=\infty$. Moreover, we have

$$
\alpha_{0}^{j}=0, \quad \alpha_{1}^{j}=\frac{1}{p_{j} B_{j}}, \quad \beta_{0}^{j}=1, \quad \gamma_{0}^{j}=a-p_{j} B_{j},
$$

for all $j$. In addition, $\beta_{i}^{j} \leq 1$ for all $i$ and $j$, and the following inclusion holds:

$$
\left\{\sigma \geq z+t\left(a-p_{j} B_{j}\right)\right\} \subset \Delta^{j}(z)
$$




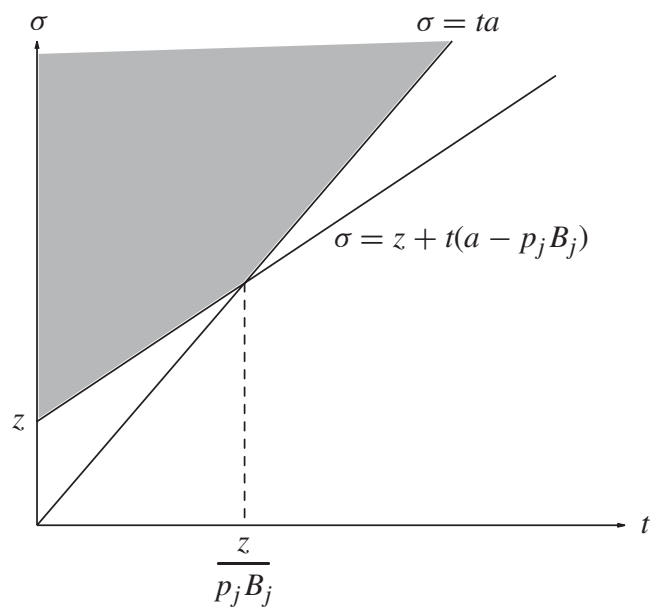

Figure 1: The first part of $\Delta^{j}(z)$.

Proof. The domain $\Delta^{j}$ may be divided into two parts, as follows:

$$
\begin{aligned}
\Delta^{j}(z) & =\left\{(\sigma, t): f^{j}(\sigma, t)>z\right\} . \\
& =\left\{\sigma>t a, \sigma>z+t\left(a-p_{j} B_{j}\right)\right\} \cup\left\{\sigma \leq t a, \sigma>a \min _{k} \frac{z+t\left(a-b_{k}\right)}{a-b_{k}+p_{j} b_{j, k}}\right\} .
\end{aligned}
$$

For the first part, we have (see Figure 1)

$$
\begin{aligned}
& \left\{\sigma>t a, \sigma>z+t\left(a-p_{j} B_{j}\right)\right\} \\
& \quad=\left\{0 \leq t<\frac{z}{p_{j} B_{j}}, \sigma>z+t\left(a-p_{j} B_{j}\right)\right\} \cup\left\{\frac{z}{p_{j} B_{j}} \leq t, \sigma>t a\right\} .
\end{aligned}
$$

For the second part, we have (see Figure 2)

$$
\left\{\sigma \leq t a, \sigma>a \min _{k} \frac{z+t\left(a-b_{k}\right)}{a-b_{k}+p_{j} b_{j, k}}\right\}=\bigcup_{k}\left\{\frac{z}{p_{j} b_{j, k}} \leq t, a \frac{z+t\left(a-b_{k}\right)}{a-b_{k}+p_{j} b_{j, k}}<\sigma \leq t a\right\} .
$$

From this, it is easy to see that the lemma holds (see Figure 3).

The inequality on the $\beta_{i}^{j}$ follows directly from the fact that $p_{j} b_{j, k} \leq b_{k}$, from which we have

$$
\frac{a}{a-b_{k}+p_{j} b_{j, k}} \geq 1
$$

Lemma 3.4. Let $X$ be a random variable such that $\bar{F}^{s} \in \delta$, let $\varepsilon_{n} \rightarrow 0$ as $n \rightarrow \infty$, and let $a(x)$ and $b(x)$ be real-valued functions such that, as $x \rightarrow \infty$,

$$
\frac{a(x)}{x} \rightarrow a, \quad \frac{b(x)}{x} \rightarrow b, \quad \text { with } 0<a<b .
$$

If $\bar{F}(x)=\mathrm{P}[X>x]$ then, for $\alpha \geq 1$ and $\beta>0$, we have

$$
\sum_{a(x) \leq n<b(x)} \mathrm{P}\left[X>\alpha x+n\left(\beta+\varepsilon_{n}\right)\right]-\sum_{a x \leq n<b x} \mathrm{P}[X>\alpha x+n \beta]=o\left(\bar{F}^{s}(x)\right) \quad \text { as } x \rightarrow \infty \text {. }
$$




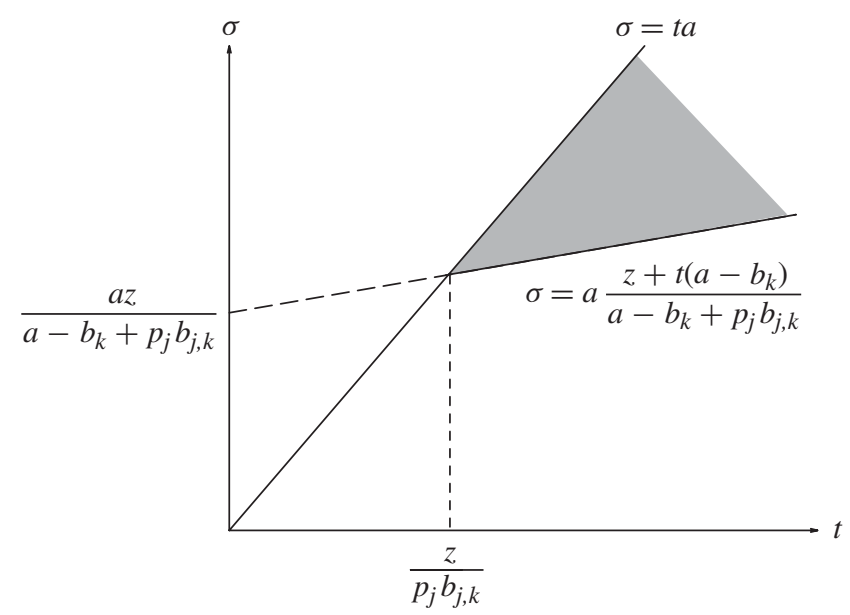

FIGURE 2: Construction of the second part of $\Delta^{j}(z)$.

Proof. For simplicity of notation, we assume that $a(x) \leq a x$ for all $x$. We have

$$
\begin{aligned}
\sum_{a(x) \leq n \leq a x} \mathrm{P}\left[X>\alpha x+n\left(\beta+\varepsilon_{n}\right)\right] & =\frac{1+\varepsilon(x)}{\beta} \int_{\alpha x+a(x) \beta}^{\alpha x+a x \beta} \bar{F}(u) \mathrm{d} u \\
& \leq \frac{1+\varepsilon(x)}{\beta} \frac{a x-a(x)}{a x} \bar{F}^{s}(\alpha x),
\end{aligned}
$$

since $\bar{F}(x)$ is nonincreasing. Hence, we have only to prove the lemma for $a(x)=a x$ and $b(x)=b x$. We have the following bound, with $\delta_{x}=\sup _{n \geq a x} \varepsilon_{n}$ :

$$
\begin{aligned}
& \sum_{a x \leq n<b x} \mathrm{P}\left[X>\alpha x+n\left(\beta+\varepsilon_{n}\right)\right]-\mathrm{P}[X>\alpha x+n \beta] \\
& \quad \leq \sum_{n} \mathrm{P}\left[X \in\left(\alpha x+n \beta, \alpha x+n\left(\beta+\delta_{x}\right)\right)\right] \\
& \quad=(1+\varepsilon(x)) \bar{F}^{s}(\alpha x)\left(\frac{1}{\beta}-\frac{1}{\beta+\delta_{x}}\right) \\
& \quad=o\left(\bar{F}^{s}(\alpha x)\right)=o\left(\bar{F}^{s}(x)\right) .
\end{aligned}
$$

Proof of Theorem 2.1. From Corollary 3.1 and Lemma 3.1, we know that the tail asymptotics of the maximal dater is linked to the quantity $S(j)$, defined by

$$
S(j)=\sum_{n \geq N_{x}} \mathrm{P}\left[Z_{[-n, 0]}>x, \bar{K}_{n}^{j}, Y_{-n}^{(j)}>x_{n}, \phi^{(j)}(-n) \leq L\right] .
$$

In the event $A_{n, x}^{j}=\bar{K}_{n}^{j} \cap\left\{Y_{-n}^{(j)}>x_{n}, \phi^{(j)}(-n) \leq L\right\}$, we have

$$
\left\{Z_{[-n, 0]}>x\right\}=\left\{f^{j}\left(Y_{-n}^{(j)}, n\right)>x-n \eta_{n}\right\}=\left\{\left(Y_{-n}^{(j)}, n\right) \in \Delta^{j}\left(x-n \eta_{n}\right)\right\} .
$$

Clearly, $\Delta^{j}(z)$ is a nonincreasing function of $z$, and we define

$$
D_{-}^{j}=: \Delta^{j}\left(x-n \varepsilon_{n}\right) \supset \Delta^{j}\left(x-n \eta_{n}\right) \supset \Delta^{j}\left(x+n \varepsilon_{n}\right)=: D_{+}^{j} .
$$




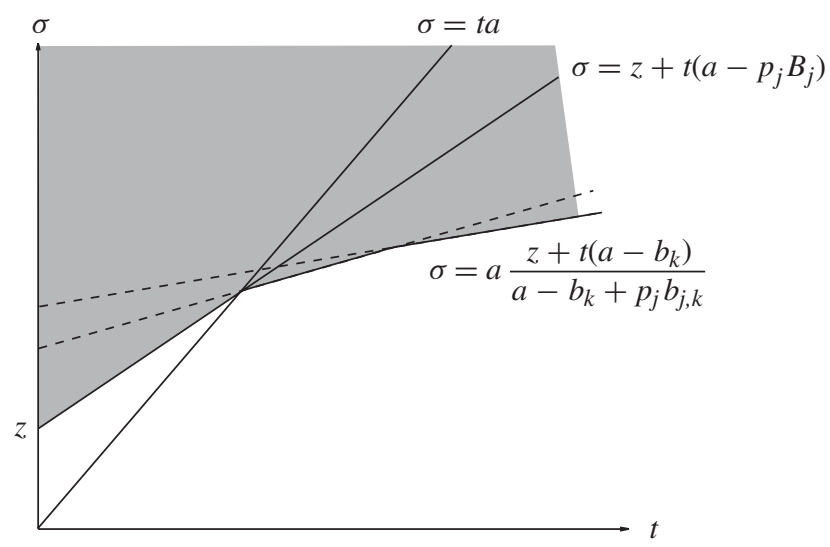

FigURE 3: Domain $\Delta^{j}(z)$.

For simplicity of notation, we write $Y^{(j)}=Y_{-n}^{(j)}$ and $\phi^{(j)}=\phi^{(j)}(-n)$. We assume, without loss of generality, that $\varepsilon_{n}$ is a decreasing sequence; hence, $\varepsilon_{n} \leq \varepsilon_{N_{x}}=\varepsilon(x)$ for $n \geq N_{x}$ and, for $n \geq N_{x}$, we have

$$
\begin{aligned}
A_{+}(n): & =\mathrm{P}\left[\left(Y^{(j)}, n\right) \in D_{+}^{j}\right] \\
& =\sum_{i=0}^{l} \mathbf{1}_{\left\{\alpha_{i}^{j}\left(x+n \varepsilon_{n}\right) \leq n<\alpha_{i+1}^{j}\left(x+n \varepsilon_{n}\right)\right\}} \mathrm{P}\left[Y^{(j)}>\frac{x}{\beta_{i}^{j}}+n \gamma_{i}^{j}+\frac{n \varepsilon_{n}}{\beta_{i}^{j}}\right] \\
& \leq \sum_{i=0}^{l} \mathbf{1}_{\left\{\alpha_{i}^{j} x \leq n<\alpha_{i+1}^{j}(x+n \varepsilon(x))\right\}} \mathrm{P}\left[Y^{(j)}>\frac{x}{\beta_{i}^{j}}+n \gamma_{i}^{j}+\frac{n \varepsilon_{n}}{\beta_{i}^{j}}\right] .
\end{aligned}
$$

Then,

$$
\begin{aligned}
\sum_{n \geq N_{x}} A_{+}(n) \leq & \sum_{N_{x} \leq n<\alpha_{1}^{j} x(1+\varepsilon(x))} \mathrm{P}\left[Y^{(j)}>x+n\left(a-p_{j} B_{j}\right)+n \varepsilon_{n}\right] \\
& +\sum_{i=1}^{l} \sum_{\alpha_{i}^{j} x \leq n<\alpha_{i+1}^{j} x(1+\varepsilon(x))} \mathrm{P}\left[Y^{(j)}>\frac{x}{\beta_{i}^{j}}+n \gamma_{i}^{j}+\frac{n \varepsilon_{n}}{\beta_{i}^{j}}\right]
\end{aligned}
$$

From Assumption 2.2, we know that $Y^{(j)}$ has an integrated tail that belongs to $\&$; therefore, as a corollary of Lemma 3.4, we have

$$
\begin{aligned}
\sum_{n \geq N_{x}} A_{+}(n)= & \sum_{0 \leq n<\alpha_{1}^{j} x} \mathrm{P}\left[Y^{(j)}>x+n\left(a-p_{j} B_{j}\right)\right] \\
& +\sum_{i=1}^{l} \sum_{\alpha_{i}^{j} x \leq n<\alpha_{i+1}^{j} x} \mathrm{P}\left[Y^{(j)}>\frac{x}{\beta_{i}^{j}}+n \gamma_{i}^{j}\right]+\varepsilon(x) \bar{F}^{s}(x) \\
= & (1+\varepsilon(x)) \sum_{i=0}^{l} \sum_{\alpha_{i}^{j} x \leq n<\alpha_{i+1}^{j} x} \mathrm{E}\left[\phi^{(j)}\right] \mathrm{P}\left[\sigma^{(j)}>\frac{x}{\beta_{i}^{j}}+n \gamma_{i}^{j}\right]+\varepsilon(x) \bar{F}^{s}(x),
\end{aligned}
$$


where the last equality follows from assumption (SE) of [4]. However, we have

$$
S(j) \leq \sum_{n \geq N_{x}} A_{+}(n)
$$

We now look at the lower bound. By the same arguments as above, we easily find that

$$
\sum_{n \geq N_{x}} A_{-}(n)=\sum_{n \geq N_{x}} A_{+}(n)+\varepsilon(x) \bar{F}^{s}(x), \quad \text { with } A_{-}(n):=\mathrm{P}\left[\left(Y^{(j)}, n\right) \in D_{-}^{j}\right] .
$$

We now show that

$$
\sum_{n \geq N_{x}} A_{-}(n)=\sum_{n \geq N_{x}} \mathrm{P}\left[\left(Y^{(j)}, n\right) \in D_{-}^{j}, A_{n, x}^{j}\right]+\varepsilon(x, L) \bar{F}^{s}(x) .
$$

Consider the difference

$$
\begin{aligned}
A_{-}(n)-\mathrm{P}\left[\left(Y^{(j)}, n\right) \in D_{-}^{j}, A_{n, x}^{j}\right] & \leq \mathrm{P}\left[\left(Y^{(j)}, n\right) \in D_{-}^{j}, \phi^{(j)}(-n)>L\right] \\
& \leq \mathrm{P}\left[Y^{(j)} \geq x+n\left(a-p_{j} B_{j}-\varepsilon_{n}\right), \phi^{(j)}(-n)>L\right],
\end{aligned}
$$

where the last inequality follows from inclusion (3.3) of Lemma 3.3. By the same kind of argument as in Corollary 3.1, we have

$$
\sum_{n \geq N_{x}} A_{-}(n)-\mathrm{P}\left[\left(Y^{(j)}, n\right) \in D_{-}^{j}, A_{n, x}^{j}\right] \leq \varepsilon(x, L) \bar{F}^{s}(x) .
$$

Hence, we have proved that, when $x \rightarrow \infty$,

$$
S(j) \sim \sum_{i=0}^{l} \sum_{\alpha_{i}^{j} x \leq n<\alpha_{i+1}^{j} x} \mathrm{E}\left[\phi^{(j)}\right] \mathrm{P}\left[\sigma^{(j)}>\frac{x}{\beta_{i}^{j}}+n \gamma_{i}^{j}\right] .
$$

Now, since this quantity is long tailed, we can use Corollary 3.1 to derive the required asymptotics for $\mathrm{P}[Z>x]$. This completes the proof.

\section{Appendix A. Proof of Corollary 3.1}

The proof is based on Proposition 3.1, which shows that

$$
\mathrm{P}[Z>x]=(1+\varepsilon(x))\left(\sum_{j=1}^{K} \sum_{n \geq N_{x}} \mathrm{P}\left[Z>x, Y_{-n}^{(j)}>x_{n}, K_{n}^{j}\right]\right) .
$$

Since $Z \geq Z_{[-n, 0]}$, we have

$$
\begin{aligned}
\mathrm{P}\left[Z>x, Y_{-n}^{(j)}>x_{n}, K_{n}^{j}\right] & \geq \mathrm{P}\left[Z_{[-n, 0]}>x, Y_{-n}^{(j)}>x_{n}, K_{n}^{j}\right] \\
& \geq \mathrm{P}\left[Z_{[-n, 0]}>x, Y_{-n}^{(j)}>x_{n}, K_{n}^{j}, \phi^{(j)}(-n) \leq L\right]
\end{aligned}
$$

and, hence,

$$
\mathrm{P}[Z>x] \geq(1+\varepsilon(x))\left(\sum_{j=1}^{K} \sum_{n \geq N_{x}} \mathrm{P}\left[Z_{[-n, 0]}>x, Y_{-n}^{(j)}>x_{n}, K_{n}^{j}, \phi^{(j)}(-n) \leq L\right]\right) .
$$


We now derive the upper bound. Take $z_{x} \rightarrow \infty$ such that $\bar{F}^{s}\left(x+z_{x}\right) \sim \bar{F}^{s}(x)$. Then, when $x \rightarrow \infty$, we have

$$
\mathrm{P}\left[Z_{[-\infty,-n-1]}<z_{x}\right]=\mathrm{P}\left[Z<z_{x}\right] \rightarrow 1 .
$$

We define $\tilde{x}=x+z_{x}$ and

$$
\tilde{K}_{n, x}^{j}=K_{n}^{j} \cap\left\{Z_{[-\infty,-n-1]} \leq z_{x}\right\}
$$

Observe that $\tilde{K}_{n, x}^{j}$ satisfies the assumptions of Proposition 3.1. By subadditivity, we have $Z \leq Z_{[-\infty,-n-1]}+Z_{[-n, 0]}$ (see [2]) and, hence,

$$
\begin{aligned}
\mathrm{P}\left[Z>\tilde{x}, \tilde{K}_{n, x}^{j}, Y_{-n}^{(j)}>\tilde{x}_{n}\right] & \leq \mathrm{P}\left[Z_{[-\infty,-n-1]}+Z_{[-n, 0]}>\tilde{x}, \tilde{K}_{n, x}^{j}, Y_{-n}^{(j)}>\tilde{x}_{n}\right] \\
& \leq \mathrm{P}\left[Z_{[-n, 0]}>x, \tilde{K}_{n, x}^{j}, Y_{-n}^{(j)}>x_{n}\right] \\
& \leq \mathrm{P}\left[Z_{[-n, 0]}>x, K_{n}^{j}, Y_{-n}^{(j)}>x_{n}\right] .
\end{aligned}
$$

We now make a partition of $\phi$ :

$$
\begin{aligned}
A(n) & =\mathrm{P}\left[Z_{[-n, 0]}>x, K_{n}^{j}, Y_{-n}^{(j)}>x_{n}\right] \\
& \leq \mathrm{P}\left[Z_{[-n, 0]}>x, K_{n}^{j}, Y_{-n}^{(j)}>x_{n}, \phi^{(j)}(-n) \leq L\right]+\mathrm{P}\left[Y_{-n}^{(j)}>x_{n}, \phi^{(j)}(-n)>L\right] \\
& =\mathrm{P}\left[Z_{[-n, 0]}>x, K_{n}^{j}, Y^{(j)}(E(-n))>x_{n}, \phi^{(j)}(-n) \leq L\right]+B(n) .
\end{aligned}
$$

We will use the following result due to Kesten (for a proof, see [1]).

Lemma A.1. Let $X \in \delta$ and let $S_{n}$ be the sum of $n$ independent copies of $X$. Then, for every $\epsilon>0$, there exists a $K(\epsilon)>0$ such that

$$
\sup _{x \geq 0} \frac{\mathrm{P}\left[S_{n}>x\right]}{\mathrm{P}[X>x]} \leq K(\epsilon)(1+\epsilon)^{n}, \quad n=1,2, \ldots
$$

Recall that $\mathrm{P}\left[\phi^{(j)}(0)=l\right]=\delta^{l}(1-\delta)$ for some $0<\delta<1$. Hence, if we take an $\epsilon$ such that $(1+\epsilon) \delta<1$, then

$$
\begin{aligned}
B(n) & =\sum_{l \geq L+1} \mathrm{P}\left[\phi^{(j)}(-n)=l\right] \mathrm{P}\left[\sum_{k=1}^{l} \sigma_{k}^{(j)}(-n)>x_{n}\right] \\
& \leq \sum_{l \geq L+1} \delta^{l}(1-\delta) K(\epsilon)(1+\epsilon)^{l} \mathrm{P}\left[\sigma^{(j)}>x_{n}\right] \\
& \leq(1-\delta) K(\epsilon) \mathrm{P}\left[\sigma^{(j)}>x_{n}\right] \frac{((1+\epsilon) \delta)^{L+1}}{1-(1+\epsilon) \delta},
\end{aligned}
$$

and we have

$$
\sum_{n \geq N_{x}} A(n) \leq \sum_{n \geq N_{x}} \mathrm{P}\left[Z>x, K_{n}^{j}, Y_{-n}^{(j)}>x_{n}, \phi^{(j)}(-n) \leq L\right]+\varepsilon(x, L) \bar{F}^{s}(x) .
$$


Since $\tilde{K}_{n, x}^{j}$ satisfies the assumptions of Proposition 3.1, we have

$$
\begin{aligned}
\mathrm{P}(Z>\tilde{x})= & \left.(1+\varepsilon(\tilde{x})) \sum_{j=1}^{K} \sum_{n \geq N_{\tilde{x}}} \mathrm{P}\left[Z>\tilde{x}, \tilde{K}_{n, x}^{j}, Y_{-n}^{(j)}>\tilde{x}_{n}\right)\right] \\
\leq & (1+\varepsilon(x)) \sum_{j=1}^{K} \sum_{n \geq N_{x}} \mathrm{P}\left[Z_{[-n, 0]}>x, K_{n}^{j}, Y_{-n}^{(j)}>x_{n}\right] \\
\leq & (1+\varepsilon(x)) \sum_{j=1}^{K} \sum_{n \geq N_{x}} \mathrm{P}\left[Z_{[-n, 0]}>x, K_{n}^{j}, Y_{-n}^{(j)}>x_{n}, \phi^{(j)}(-n) \leq L\right] \\
& +\varepsilon(x, L) \bar{F}^{s}(x) .
\end{aligned}
$$

Hence, we have shown, in the notation of Lemma A.1, that

$$
\begin{aligned}
\mathrm{P}[Z>x] & \geq(1+\varepsilon(x)) G(x), \\
\mathrm{P}\left[Z>x+z_{x}\right] & \leq(1+\varepsilon(x)) G(x)+\varepsilon(x, L) \bar{F}^{s}(x) .
\end{aligned}
$$

From these inequalities, we can directly derive (3.1). If $G$ is long tailed, we can choose $z_{x} \rightarrow \infty$ such that $G\left(x+z_{x}\right) \sim G(x)$ and $\bar{F}^{s}\left(x+z_{x}\right) \sim \bar{F}^{s}(x)$, and the last statement of Corollary 3.1 follows.

\section{References}

[1] Athreya, K. B. And Ney, P. E. (1972). Branching Processes. Springer, Berlin.

[2] Baccelli, F. AND Foss, S. (1994). Ergodicity of Jackson-type queueing networks. Queuing Systems Theory Appl. 17, 5-72.

[3] Baccelli, F. And Foss, S. (1995). On the saturation rule for the stability of queues. J. Appl. Prob. 32, 494-507.

[4] Baccelli, F. And Foss, S. (2004). Moments and tails in monotone-separable stochastic networks. Ann. Appl. Prob. 14, 612-650.

[5] Embrechts, P., Goldie, C. and Veraverbeke, N. (1979). Subexponentiality and infinite divisibility. Z. Wahrscheinlichkeitsth. 49, 335-347.

[6] Goldie, C. M. And KlüPPElberg, C. (1998). Subexponential distributions. In A Practical Guide to Heavy Tails, eds R. J. Adler, R. E. Feldman and M. S. Taqqu, Birkhäuser, Boston, MA, pp. 435-459.

[7] Lelarge, M. (2005). Fluid limit of generalized Jackson queueing networks with stationary and ergodic arrivals and service times. J. Appl. Prob. 42, 491-512. 